\title{
ADDITION THEOREMS FOR SPHERICAL WAVE FUNCTIONS*
}

\author{
BY \\ SEYMOUR STEIN \\ Sylvania Electronic Systems, Waltham, Mass.
}

\begin{abstract}
Addition theorems are described for spherical vector wave functions, under both rotations and translations of the coordinate system. These functions are the characteristic solutions in spherical coordinates of the vector wave equation, such as occurs in electromagnetic problems. The vector wave function addition theorems are based on corresponding theorems for the spherical scalar wave functions. The latter are reviewed and discussed.
\end{abstract}

1. Introduction. Boundary-value problems often involve several bodies or more than one important reference point. It can then be very convenient to be able to expand the field solutions in alternative forms, each form referring to a different specific coordinate set describing the same space. The connections between the alternate representations are provided by "addition theorems", i.e., formulas for the expansion of the basis set of one representation in terms of the basis set of another. In general, the completeness of such basis sets indicates immediately the existence of these expansions, although their specification may not be obvious.

The results discussed here were motivated by a problem in electromagnetic scattering in which exactly such a need arose. The basis sets considered are the so-called spherical vector wave functions, i.e., characteristic vector field solutions of the vector wave equation, based on a conventional separation of variables in spherical coordinates $(R, \theta, \phi)$. The set of vector wave functions can be described by using as potentials the set of scalar wave functions (the characteristic solutions to the scalar wave equation). Addition theorems for the latter are however available in the literature. In this paper, these are cited and briefly discussed, and extended to provide the desired general addition theorems for vector wave functions. The only other known calculations of these addition theorems, derived for specific low orders of vector wave functions by direct but laborious procedures, and applied to some particular problems, appears in Ament [1].

2. Characterization of the vector wave functions. The electric and magnetic fields in a source-free homogeneous medium are divergenceless, and each satisfies a vector wave equation of form

$$
\nabla \times \nabla \times \mathbf{A}-k^{2} \mathbf{A}=\mathbf{0},
$$

$k$ being a constant for the medium. It is well-known (Stratton, [2]) that independent solutions of this equation can be constructed as

$$
\begin{aligned}
& \mathbf{M}_{m n}=\nabla u_{m n} \times \mathbf{R}, \\
& \mathbf{N}_{m n}=\frac{1}{k} \nabla \times \mathbf{M}_{m n},
\end{aligned}
$$

*Received December 26, 1959; revised manuscript received April 4, 1960. This research was carried out at Hermes Electronics Co., Cambridge, Mass. and was supported by the Defense Atomic Support Agency through the Office of Naval Research under Contract Nonr-2632(00). 
with the added interrelation $\mathbf{M}_{m n}=1 / k \nabla \times \mathbf{N}_{m n}$, where $\mathbf{R}$ is a position vector from the origin 0 , and the potentials $u_{m n}$ are a corresponding complete set of solutions of the scalar wave equations

$$
\nabla^{2} u+k^{2} u=0 .
$$

In spherical coordinates, such a set of characteristic solutions are given by

$$
u_{m n}=z_{n}(k R) P_{n}^{m}(\cos \theta) \exp (i m \phi),-n \leq m \leq n, \quad 0 \leq n<\infty .
$$

Here $z_{n}(k R)$ is generically any spherical Bessel function, say as defined by Stratton 3]. The associated Legendre function $P_{n}^{m}(\cos \theta)$ will also be taken following Stratton [4], as

$$
P_{n}^{m}(x)=\frac{\left(1-x^{2}\right)^{m / 2}}{2^{n} n !} \frac{d^{n+m}}{d x^{n+m}}\left(x^{2}-1\right)^{n}, \quad|m| \leq n .
$$

The latter differs by a $(-)^{m}$ factor from one common alternative definition.

The specific forms for the vector wave functions are

$$
\begin{aligned}
& \mathbf{M}_{m n}=z_{n}(k R)\left[\mathbf{i}_{2} \frac{i m P_{n}^{m}(\cos \theta)}{\sin \theta} \exp (i m \phi)-\mathbf{i}_{3} \exp (i m \phi) \frac{d}{d \theta} P_{n}^{m}(\cos \theta)\right], \\
\mathbf{N}_{m n}= & \mathbf{i}_{1} \frac{z_{n}(k R)}{k R} n(n+1) P_{n}^{m}(\cos \theta) \exp (i m \phi) \\
+ & \frac{1}{k R} \frac{d}{d R}\left[R z_{n}(k R)\right]\left[\mathbf{i}_{2} \exp (i m \phi) \frac{d}{d \theta} P_{n}^{m}(\cos \theta)+\mathbf{i}_{3} \frac{i m P_{n}^{m}(\cos \theta)}{\sin \theta} \exp (i m \phi)\right],
\end{aligned}
$$

where $\dot{i}_{1}, \dot{i}_{2}, \dot{i}_{3}$ are unit vectors in the directions of increasing $R, \theta, \phi$ respectively. It is useful to note that for $n=m=0$, we have $\mathbf{M}_{00}=\mathbf{N}_{00}=0$.

We note that Maxwell's equations for harmonic time-dependence exp $(-i \omega t)$, and for sourceless regions are

$$
\begin{aligned}
\nabla \times \mathbf{E} & =i \omega \mu \mathbf{H}, \\
\nabla \times \mathbf{H} & =-i \omega \epsilon \mathbf{E},
\end{aligned}
$$

where $\omega^{2} \mu \epsilon \equiv k^{2}$. It follows from Eq. (2) that to an $E$-field contribution of the form $\mathbf{M}_{m n}$ there is associated exactly an $H$-field term, $(k / i \omega \mu) \mathbf{N}_{m n}$; similarly, to an $E$-field contribution $\mathbf{N}_{m n}$, there is associated an $H$-field term $(k / i \omega \mu) M_{m n}$. Furthermore, the detailed forms in Eqs. (6) show that $\mathbf{M}_{m n}$ has no radial component, and hence all radial field components must be represented by the $\mathbf{N}_{m n}$ solely. Thus, in fact, it is common to distinguish in electromagnetic theory two types of modes: the $H$-type in which only the magnetic field has a radial component, and the $E$-type in which only the electric field has a radial component. It is clear that $H$-type modes have $\mathbf{E}$ represented by the $\mathbf{M}_{m n}$ and $\mathbf{H}$ by the $\mathbf{N}_{m n}$, and the $E$-type modes vice-versa. For any general excitation, both types of modes may be present, and so the general field summations would be of the form

$$
\begin{aligned}
& \mathbf{E}=\sum_{n=1}^{\infty} \sum_{m=-n}^{\infty}\left[A_{m n} \mathbf{M}_{m n}+B_{m n} \mathbf{N}_{m n}\right], \\
& \left.\mathbf{H}=(k / i \omega \mu) \sum_{n=1}^{\infty} \sum_{m=-n}^{\infty}{ }_{L} A_{m n} \mathbf{N}_{m n}+B_{m n} \mathbf{M}_{m n}\right] .
\end{aligned}
$$

Finally we note that if we make either a translation or rotation, or both, of the coordinates, a new set of scalar and vector wave functions can be analogously defined 
with respect to the new coordinates. Since any one of the previous vector wave functions defines a perfectly valid vector field, it must be expandable over the new set. That is, under coordinates rotations and/or translations, there must exist addition theorems of the form

$$
\begin{aligned}
& \mathbf{M}_{\mu \nu}(R, \theta, \phi) \\
& \quad=\sum_{n=1}^{\infty} \sum_{m=-n}^{n}\left[C(\mu, \nu \mid m, n) \mathbf{M}_{m n}\left(R^{\prime}, \theta^{\prime}, \phi^{\prime}\right)+D(\mu, \nu \mid m, n) \mathbf{N}_{m n}\left(R^{\prime}, \theta^{\prime}, \phi^{\prime}\right)\right], \\
& \mathbf{N}_{\mu \nu}(R, \theta, \phi) \\
& \quad=\sum_{n=1}^{\infty} \sum_{m=-n}^{n}\left[C(\mu, \nu \mid m, n) \mathbf{N}_{m n}\left(R^{\prime}, \theta^{\prime}, \phi^{\prime}\right)+D(\mu, \nu \mid m, n) \mathbf{M}_{m n}\left(R^{\prime}, \theta^{\prime}, \phi^{\prime}\right)\right],
\end{aligned}
$$

where $\left(R^{\prime}, \theta^{\prime}, \phi^{\prime}\right)$ is the new set of spherical coordinates. In the above, we may note that the second equation follows directly from the first by utilizing the basic definitions in Eqs. (2), and noting additionally that the curl operator is invariant of the choice of coordinate system. We note also that the coefficients will of course contain implicitly the geometric parameters which relate the two coordinate systems.

We will describe the representations for rotations and translations separately. A coordinate change involving both types will be a "multiplication" of the two operations.

3. Rotation of coordinates. It is extremely simple to describe the addition theorems for $\mathbf{M}_{m n}$ and $\mathbf{N}_{m n}$ under coordinate rotations.

We assume a second set of coordinates $\left(R, \theta^{\prime}, \phi^{\prime}\right)$ centered at 0 , but with reference axes rotated with respect to the original $(R, \theta, \phi)$ system. Note that the radial coordinate is invariant under rotation. In Appendix 1, we characterize such a rotation, and state the known addition theorem for the spherical scalar wave functions, which is in the form*

$$
u_{\mu n}(R, \theta, \phi)=\sum_{m=-n}^{n} \beta(\mu, m, n) u_{m n}^{\prime}\left(R, \theta^{\prime}, \phi^{\prime}\right) .
$$

But the vector operator $\nabla$ is defined independently of the coordinate system, and due to the common origin, the same $\mathbf{R}$ is the position vector for both. It follows immediately by using Eq. (10) and the basic definitions that the vector wave functions are related by

$$
\mathbf{M}_{\mu n}(R, \theta, \phi)=\nabla u_{\mu n}(R, \theta, \phi) \times \mathbf{R}=\sum_{m=-n}^{n} \beta(\mu, m, n) \mathbf{M}_{m n}^{\prime}\left(R, \theta^{\prime}, \phi\right)
$$

and this is the complete specification for coordinate rotations, of the general characterization in Eq. (9).

4. Translation of coordinate origin. Suppose a second origin of coordinates is taken at a point $0^{\prime}$, whose coordinates are $\left(R_{0}, \theta_{0}, \phi_{0}\right)$ with respect to the first. The set of spherical coordinates $\left(R^{\prime}, \theta^{\prime}, \phi^{\prime}\right)$ is introduced with respect to $0^{\prime}$, such that the polar axis, $\theta^{\prime}=0$, and the azimuth axis, $\phi^{\prime}=0$, are respectively parallel to the corresponding axes, $\theta=0$ and $\phi=0$. This is then a rigid translation of the coordinate system. In Appendix 2, we state the known addition theorem for the spherical scalar wave function under such translation, in the form

*The prime notation on the $u_{m n}^{\prime}$ is to emphasize the coordinate set with respect to which the potential is defined. We will utilize primes for this purpose throughout; under no circumstances should they be interpreted here as indicating differentiations. 


$$
u_{\mu \nu}(R, \theta, \phi)=\sum_{n=0}^{\infty} \sum_{m=-n}^{n} \alpha(\mu, \nu \mid m, n) \mu_{m n}^{\prime}\left(R^{\prime}, \theta^{\prime}, \phi^{\prime}\right) .
$$

(We note from Appendix 2 that, depending on the value of $R$ relative to $R_{0}$, the $u_{m n}^{\prime}$ may involve a spherical Bessel function $z_{n}^{\prime}(k R)$ which is generically not of the same type as the $z_{\nu}(k R)$ involved in $u_{\mu \nu}$. This distinction will not be important in the derivation below, which involves only the local properties of $u_{m n}^{\prime}$, whichever Bessel function type is contained.)

Since the grad operation is invariant of coordinate system, we can immediately write*

$$
\begin{aligned}
\mathbf{M}_{\mu \nu}(R, \theta, \phi) & =\nabla u_{\mu \nu} \times \mathbf{R} \\
& =\sum_{n=0}^{\infty} \sum_{m=-n}^{n} \alpha(\mu, \nu \mid m, n)\left[\nabla u_{m n}^{\prime} \times \mathbf{R}\right] .
\end{aligned}
$$

Our problem therefore is to expand terms of the form $\nabla u_{m n}^{\prime} \times \mathbf{R}$ in terms of $\mathbf{M}_{m n}^{\prime}$ and $\mathbf{N}_{m n}^{\prime}$ vector wave functions.

If $\mathbf{R}_{0}$ is the position vector of the second origin, $0^{\prime}$, with respect to the first origin, 0 , we can further write

$$
\mathbf{R}=\mathbf{R}_{0}+\mathbf{R}^{\prime}
$$

But then

$$
\nabla u_{m n}^{\prime} \times \mathbf{R}=\left(\nabla u_{m n}^{\prime} \times \mathbf{R}_{0}\right)+\left(\nabla u_{m n}^{\prime} \times \mathbf{R}^{\prime}\right)
$$

and we immediately recognize the last term as simply

$$
\nabla u_{m n}^{\prime} \times \mathbf{R}^{\prime}=\mathbf{M}_{m n}^{\prime} \text {. }
$$

Hence it remains only to determine an expansion for $\nabla u_{m n}^{\prime} \times \mathbf{R}_{0}$.

The simplest approach to the latter has appeared to be to identify first the part which is represented by the $\mathbf{N}_{m n}^{\prime}$ functions, since this involves matching only the vector components in the $i_{1}^{\prime}$ direction. That is, using Eq. (6b), we seek an expansion of the form

$$
\begin{aligned}
\mathbf{i}_{1}^{\prime} \cdot \nabla u_{m n}^{\prime} \times \mathbf{R}_{0} & =\sum_{p=1}^{\infty} \sum_{a=-p}^{p} A_{a \mathbf{p}_{1}} \cdot \mathbf{N}_{a p}^{\prime}, \\
& =\sum_{p=1}^{\infty} \sum_{a=-p}^{p} A_{a p} \frac{z_{p}\left(k R^{\prime}\right)}{k R^{\prime}} p(p+1) P_{p}^{a}\left(\cos \theta^{\prime}\right) \exp \left(i q \phi^{\prime}\right) .
\end{aligned}
$$

It may readily be ascertained that a fixed vector field of magnitude and direction equal to $R_{0}$ is represented at the general point $\left(R^{\prime}, \theta^{\prime}, \phi^{\prime}\right)$ by components

$$
\begin{aligned}
& \mathbf{R}_{0} \cdot \dot{\mathbf{i}}_{1}^{\prime}=R_{0}\left[\sin \theta_{0} \sin \theta^{\prime} \cos \left(\phi_{0}-\phi^{\prime}\right)+\cos \theta_{0} \cos \theta^{\prime}\right], \\
& \mathbf{R}_{0} \cdot \dot{\mathbf{i}}_{2}^{\prime}=R_{0}\left[\sin \theta_{0} \cos \theta^{\prime} \cos \left(\phi_{0}-\phi^{\prime}\right)-\cos \theta_{0} \sin \theta^{\prime}\right], \\
& \mathbf{R}_{0} \cdot \dot{i}_{3}^{\prime}=R_{0}\left[\sin \theta_{0} \sin \left(\phi_{0}-\phi^{\prime}\right)\right] .
\end{aligned}
$$

Most of the derivation then consists in utilizing this result, along with trigonometric identities, and well-known Legendre and Bessel function recurrence relations. The algebraic manipulations are tedious but reproducible, and for the sake of brevity, the algebraic details will be omitted here.

*In writing Eq. (12), we can recall explicitly that we are only interested in the form of expansion for $\nu \geq 1,|\mu| \leq \nu$; this is implicit throughout the remainder of the discussion. 
For the radial part, comparison of the expansions on the two sides of Eq. (16) yields

$$
\begin{gathered}
n=m=0: \dot{i}_{1}^{\prime} \cdot \nabla u_{00}^{\prime} \times \mathbf{R}_{0}=0, \\
n \geq 1: \mathbf{i}_{1}^{\prime} \cdot \nabla u_{m n}^{\prime} \times \mathbf{R}_{0}=\frac{k R_{0}}{n(n+1)} \mathbf{i}_{1}^{\prime} \cdot\left\{i m \cos \theta_{0} \mathbf{N}_{m n}^{\prime}\right. \\
-\frac{i}{2} \sin \theta_{0} \exp \left(-i \phi_{0}\right) \mathbf{N}_{m+1, n}^{\prime} \\
\left.-\frac{i}{2} \sin \theta_{0} \exp \left(i \phi_{0}\right)(n+m)(n-m+1) \mathbf{N}_{m-1, n}^{\prime}\right\} .
\end{gathered}
$$

This completely identifies the radial component, and hence all the possible $\mathbf{N}_{m n}^{\prime}$ which enter the expansion of $\nabla u_{m n}^{\prime} \times R_{0}$.

If we next consider the $\mathbf{i}_{2}^{\prime}$ or $\mathbf{i}_{3}^{\prime}$ component of $\nabla u_{m n}^{\prime} \times \mathbf{R}_{0}$, subtracting off the corresponding component of the vector whose radial component is indicated on the $R H S$ of Eq. (18), we can (after much manipulation) identify the remainder in terms of components of $\mathbf{M}_{m n}^{\prime}$. The result may be summarized as follows:

$n=m=0$ :

$\nabla u_{00}^{\prime} \times \mathbf{R}_{0}=k R_{0} \cos \theta_{0} \mathbf{M}_{01}^{\prime}+\frac{k R_{0}}{2} \sin \theta_{0} \exp \left(-i \phi_{0}\right) \mathbf{M}_{11}^{\prime}-k R_{0} \sin \theta_{0} \exp \left(i \phi_{0}\right) \mathbf{M}_{-1,1}^{\prime}$

and for $n \geq 1$

$$
\begin{aligned}
\nabla u_{m n}^{\prime} \times \mathbf{R}_{0} & -\frac{k R_{0}}{n(n+1)}\left\{i m \cos \theta_{0} \mathbf{N}_{m n}^{\prime}-\frac{i}{2} \sin \theta_{0} \exp \left(-i \phi_{0}\right) \mathbf{N}_{m+1, n}^{\prime}\right. \\
& \left.-\frac{i}{2} \sin \theta_{0} \exp \left(i \phi_{0}\right)(n+m)(n-m+1) \mathbf{N}_{m-1, n}^{\prime}\right\} \\
& =\mathbf{M}_{m n}^{\prime}+\frac{k R_{0}}{2 n+1}\left\{\cos \theta_{0}\left[\frac{n+m}{n} \mathbf{M}_{m, n-1}^{\prime}+\frac{n-m+1}{n+1} \mathbf{M}_{m, n+1}^{\prime}\right]\right. \\
& +\frac{\sin \theta_{0} \exp \left(-i \phi_{0}\right)}{2}\left[-\frac{1}{n} \mathbf{M}_{m+1, n-1}^{\prime}+\frac{1}{n+1} \mathbf{M}_{m+1, n+1}^{\prime}\right] \\
& +\frac{\sin \theta_{0} \exp \left(i \phi_{0}\right)}{2}\left[\frac{(n+m)(n+m-1)}{n} \mathbf{M}_{m-1, n-1}^{\prime}\right. \\
& \left.\left.-\frac{(n-m+1)(n-m+2)}{n+1} \mathbf{M}_{m-1, n+1}^{\prime}\right]\right\} .
\end{aligned}
$$

We may note that Eq. (19a) is subsumed in Eq. $19 \mathrm{~b}$ if it is assumed as a definition that $\mathbf{M}_{p, n-1}=0$ when $n<0$ and that all terms in $\mathbf{N}_{p n}$ are $\equiv 0$ when $n=0$; we will use these conventions in the last result below.

We can refer back now to the general form of expansion in Eq. (9), and summarize Eqs. (11, 12, 14, 15 and 19). Further, making some obvious appropriate changes in certain of the summation indices, we find that the addition theorem under rigid coordinate translation has the form

$$
\begin{aligned}
\mathbf{M}_{\mu \nu}(R, \theta, \phi)=\sum_{n=1}^{\infty} \sum_{m=-n}^{n}\left[C(\mu, \nu \mid m, n) M_{m n}^{\prime}\left(R^{\prime}, \theta^{\prime}, \phi^{\prime}\right)\right. & \\
& \left.+D(\mu, \nu \mid m, n) N_{m n}^{\prime}\left(R^{\prime}, \theta^{\prime}, \phi^{\prime}\right)\right],
\end{aligned}
$$


where

$$
\begin{aligned}
& C(\mu, \nu \mid m, n)=\alpha(\mu, \nu \mid m, n)+\frac{k R_{0} \cos \theta_{0}}{2 n+3} \frac{n+m+1}{n+1} \alpha(\mu, \nu \mid m, n+1) \\
& +\frac{k R_{0} \cos \theta_{0} n-m}{(2 n-1)} \alpha(\mu, \nu \mid m, n-1) \\
& -\frac{k R_{0} \sin \theta_{0} \exp \left(-i \phi_{0}\right)}{2(2 n+3)(n+1)} \alpha(\mu, \nu \mid m-1, n+1) \\
& +\frac{k R_{0} \sin \theta_{0} \exp \left(-i \phi_{0}\right)}{2(2 n-1) n} \alpha(\mu, \nu \mid m-1, n-1) \\
& +\frac{k R_{0} \sin \theta_{0} \exp \left(i \phi_{0}\right)}{2(2 n+3)} \frac{(n+m+2)(n+m+1)}{n+1} \alpha(\mu, \nu \mid m+1, n+1) \\
& -\frac{k R_{0} \sin \theta_{0} \exp \left(i \phi_{0}\right)}{2(2 n-1)} \frac{(n-m-1)(n-m)}{n} \alpha(\mu, \nu \mid m+1, n-1), \\
& \quad-\frac{i k R_{0} \cos \theta_{0}}{n(n+1)} m \alpha(\mu, \nu \mid m, n) \\
& \quad-\frac{i k R_{0} \sin \theta_{0} \exp \left(-i \phi_{0}\right)}{2 n(n+1)} \alpha(\mu, \nu \mid m-1, n) \\
& \quad-\frac{i k R_{0} \sin \theta_{0} \exp \left(i \phi_{0}\right)}{2 n(n+1)}(n+m+1)(n-m) \alpha(\mu, \nu \mid m+1, n) .
\end{aligned}
$$

In the above $\alpha(\mu, \nu \mid m, n)$ is taken to vanish whenever $|m|>n$ (see, e.g., Eqs. (A2-4 and A2-2) of Appendix 2).

It would of course be helpful if the expressions for $C(\mu, \nu \mid m, n)$ and $D(\mu, \nu \mid m, n)$ could be reduced, or could be shown to satisfy useful recurrence formulas. One might expect that use of formulas such as indicated in Appendix 2 might lead to such a reduction. However, to date, although some suggestive results have been noted, no clear cut simplifications over the formulas above have been found.

Acknowledgment. The assistance of Mr. George Foglesong, presently at MIT, in compiling some of the Appendix material, is gratefully acknowledged.

\section{Appendix I}

\section{Addition Theorems for Scalar Spherical Wave Functions}

\section{Under Coordinate Rotations}

These theorems are well known (especially in applications in quantum mechanics) as a special case of the study of the 3-dimensional rotation group. Various forms of the derivations, in largely non-group theoretical terms, appear in [5-9]. Some group theoretical derivations appear in [10], along with general recurrence formulas for the coefficients, and tables covering lower-order cases. We have found it convenient, however, to refer primarily to the forms common in quantum mechanics, such as presented by Edmonds [11]. In terms of the Euler angles of rotation, the addition theorem appears as

$$
Y_{j m}(\theta, \phi)=\sum_{m^{\prime}=-j}^{i} Y_{i m^{\prime}}\left(\theta^{\prime}, \phi^{\prime}\right) D_{m^{\prime} m}^{(i)}(\alpha \beta \gamma)
$$


with the relation to our notation [12] of

$$
Y_{i m}(\theta, \phi)=(-)^{m}\left[\left(\frac{2 j+1}{4 \pi}\right) \frac{(j-m) !}{(j+m) !}\right]^{1 / 2} P_{i}^{m}(\cos \theta) \exp (i m \phi) .
$$

The coordinates $\theta, \phi$, are the angle coordinates of a point in space defined in the usual manner with respect to one set of cartesian axes, and $\theta^{\prime}, \phi^{\prime}$ are similarly the coordinates of the same point with respect to a new set of axes, the latter set of axes being obtained by a rigid-body rotation of the first set of axes through the Euler angle $\alpha, \beta, \gamma$. The convention used by Edmonds [13] is that the angles $\alpha, \beta, \gamma$ are positive in the sense of rotation of a right-handed screw in the right-handed frame of axes. The coefficients $D_{m, m}^{(i)}(\alpha \beta \gamma)$ are the matrix elements of a unitary transformation, and are given by

$$
D_{m^{\prime} m}^{(i)}(\alpha \beta \gamma)=\left[\exp i m^{\prime} \alpha\right] d_{m^{\prime} m}^{(i)}(\beta)[\exp i m \gamma]
$$

where

$$
\begin{aligned}
d_{m^{\prime} m}^{(i)}(\beta)=\left[\frac{\left(j+m^{\prime}\right) !\left(j-m^{\prime}\right) !}{(j+m) !(j-m) !}\right]^{1 / 2} & \sum_{\sigma}\left(\begin{array}{c}
j+m \\
j-m^{\prime}-\sigma
\end{array}\right)\left(\begin{array}{c}
j-m \\
\sigma
\end{array}\right) \\
& (-)^{j-m^{\prime}-\sigma}\left(\cos \frac{\beta}{2}\right)^{2 \sigma+m^{\prime}+m}\left(\sin \frac{\beta}{2}\right)^{2 i-2 \sigma-m^{\prime}-m} .
\end{aligned}
$$

In terms of our form (Eq. 10), we note that both sides of Eq. (A1-1) involve surface harmonics of the same order, $j$, and hence it is a trivial step to introduce additional radial wave function factors $z_{j}(k R)$ on both sides to obtain a relation on total wave functions. Using our definition of the wave function (Eq. 4) we can rewrite Eq. (A1-1) in the form

$$
u_{\mu n}(R, \theta, \phi)=\sum_{m=-n}^{n} \beta(\mu, m, n) u_{m n}\left(R^{\prime}, \theta^{\prime}, \phi^{\prime}\right),
$$

where, using also Eq. (A1-2),

$$
\beta(\mu, m, n)=(-)^{m+\mu}\left[\frac{(n+\mu) !}{(n-\mu) !} \frac{(n-m) !}{(n+m) !}\right]^{1 / 2} D_{m \mu}^{(n)}(\alpha \beta \gamma) .
$$

Several useful sets of recurrence formulas are available for these coefficients, as special cases of general transformation laws [14-17]. but are omitted here, for brevity.

\section{Appendix II}

Addition Theorems for the Scalar Spherical Wave Functions Under Coordinate Translations

1. A restatement of the Friedman-Russek result. A derivation of the translational addition theorem for scalar spherical wave functions has been given by Friedman and Russek [18]. However, they use a normalized Legendre function such that $P_{n}^{-m}(\cos \theta)=$ $P_{n}^{m}(\cos \theta)$; and they appear to fail to note that their final statement of the addition theorem depends on the premise that $|m+\mu|=|m|+|\mu|$ where $m, \mu$ are azimuthal indices of associated Legendre functions. Since the latter is not correct when $m$ and $\mu$ are of opposite sign, we cite here the basically correct form of their result, but give the correct values for the coefficients. In addition Friedman and Russek only identify these coefficients as the values of certain integrals over triple products of Legendre functions, the values of which in turn are found in the literature as rather complicated 
sums. Instead, we point out that these coefficients are quite well known in the study of angular momentum in quantum mechanics, being in essence the vector-coupling coefficients (or Wigner $3-j$ symbols) which describe the matrix elements of a unitary transformation between a product-state representation and a compound-state representation in a two-particle system. With this recognition, we are able to draw upon a large body of known information for useful computational forms and recurrence relations. Finally, we will correct below some errors of notation in the original results, as well as present a more useful form for one set of the results.

To begin with, let us recall that our basic definition of the Legendre function (Eq. 5) can be regarded [19] as valid for negative $m$ as well as positive, in the range $-n \leq m \leq n$ (we have in fact so used $P_{n}^{m}$ in the text).

With this in mind, the basic result derived by Friedman and Russek in [18] can be stated as follows:

In an $(R, \theta, \phi)$ coordinate system, let the point $0^{\prime}$ at $\left(R_{0}, \theta_{0}, \phi_{0}\right)$ be taken as the origin of a second coordinate system with coordinates $\left(R^{\prime}, \theta^{\prime}, \phi^{\prime}\right)$, and oriented so that a rigid body translation [by the vector $R_{0}\left(R_{0}, \theta_{0}, \phi_{0}\right)$ ] takes one system into the other. Then we have the addition theorem, ${ }^{*}$ valid for $\nu \geq 0,-\nu \leq \mu \leq \nu$, and where $z_{\nu}$ is any spherical cylinder function,

$z_{\nu}(k R) P_{\nu}^{\mu}(\cos \theta) \exp (i \mu \phi)=i^{-\nu} \sum_{n=0}^{\infty} \sum_{m=-n}^{n} \sum_{p}\left\{i^{n+p}(2 n+1)(-)^{m} a(\mu, m \mid p, \nu, n)\right.$
$\cdot z_{p}\left(k r_{>}\right) P_{p}^{\mu+m}\left(\cos \theta_{>}\right) \exp \left[i(\mu+m) \phi_{>}\right]$
$\left.\cdot j_{n}\left(k r_{<}\right) P_{n}^{-m}\left(\cos \theta_{<}\right) \exp \left(-i m \phi_{<}\right)\right\}$,

where

$$
\left\{\begin{array}{ll}
r_{>}=R^{\prime} & r_{<}=R_{0} \\
\theta_{>}=\theta^{\prime} & \theta_{<}=\theta_{0} \\
\phi_{>}=\phi^{\prime} & \phi_{<}=\phi_{0}
\end{array}\right\} \text { when } R^{\prime} \geq R_{0},
$$

and

$$
\left\{\begin{array}{ll}
r_{>}=R_{0} & r_{<}=R^{\prime} \\
\theta_{>}=\theta_{0} & \theta_{<}=\theta^{\prime} \\
\phi_{>}=\phi_{0} & \phi_{<}=\phi^{\prime}
\end{array}\right\} \text { when } R^{\prime} \leq R_{0} .
$$

The sum over $p$ is over all the values

$$
p=n+\nu, \quad n+\nu-2, \quad n+\nu-4, \cdots
$$

(but no lower than $|n-\nu|)$, for which the coefficient $a(\mu, m \mid p, \nu, n)$ is non-vanishing. The latter coefficient is defined by the expansion

$$
P_{n}^{m}(\cos \theta) P_{\nu}^{\mu}(\cos \theta)=\sum_{p} a(\mu m, \mid p, \nu, n) P_{p}^{m+\mu}(\cos \theta)
$$

${ }^{*}$ The introduction and use below of the notation $\theta_{>}, \theta_{<}, \phi_{>}, \phi_{<}$serves to correct a rather obvious error in the Friedman-Russek paper, in the writing of their Eqs. (19) and (21); the error is obvious from their derivation which involves the parameters only in the quantities $r \cos \gamma$ and $r_{0} \cos \gamma_{0}$. 
which is known to exist in exactly such a form on the basis of spherical harmonic expansion theorems, with $n+\nu \geq p \geq|n-\nu|$. It is apparent from the orthonormality properties of the $p_{p}^{a}(\cos \theta)$ that $a(\mu, m \mid p, \nu, n)$ can be determined from Eq. (A2-2) in terms of an integral over a special triple product of associated Legendre functions. Such integrals have been investigated in the literature of quantum mechanics, and Friedman and Russek cite one such evaluation, a rather complicated sum involving multitudinous factorials.

Now, it is desirable to rewrite the result, Eq. (A2-1), in a more useful form, namely the form Eq. (11) in which we have used the addition theorem in the text

$$
u_{\mu \nu}(R, \theta, \phi)=\sum_{n=0}^{\infty} \sum_{m=-n}^{n} \alpha(\mu, \nu \mid m, n) u_{m n}^{\prime}\left(R^{\prime}, \theta^{\prime}, \phi^{\prime}\right) .
$$

A comparison with Eq. (A2-1) shows that for $R^{\prime} \leq R_{0}$, we have exactly such a form where

$$
\alpha(\mu, \nu \mid m, n)=i^{-\nu+n}(2 n+1)(-)^{m} \sum_{p} i^{p} a(\mu,-m \mid p, \nu, n) u_{p}^{\mu-m}\left(R_{0}, \theta_{0}, \phi_{0}\right) .
$$

We note that in these equations for $R^{\prime} \leq R_{0}$, the $u_{m n}^{\prime}\left(R^{\prime}, \theta^{\prime}, \phi^{\prime}\right)$ contain explicitly a $j_{n}\left(k R^{\prime}\right)$ dependence, no matter what the form of the spherical cylinder function in $u_{\mu \nu}(R, \theta, \phi) ;$ and on the other hand, the $u_{p}^{\mu-m}\left(R_{0}, \theta_{0}, \phi_{0}\right)$ contains exactly the same type of dependence $z_{p}\left(k R_{0}\right)$ as is contained in $u_{\mu \nu}(R, \theta, \phi)$ in the form $z_{\nu}(k R)$.

However, for $R^{\prime} \geq R_{0}$, the $R^{\prime}$ variations in the form of Eq. (A2-1) go into the $u_{p}^{\mu-m}$, and in order to obtain a form like Eq. (A2-3), it is necessary to interchange the orders of summation. That such an interchanged form must be available is obvious, since the result is then exactly the one expected from the usual technique of expanding an arbitrary function of $(R, \theta, \phi)$ in terms of a new coordinate origin and new coordinate set. We accomplish the interchange by noting that in Eq. (A2-1), $p$ eventually takes on all values between 0 and $\infty$. Furthermore, the formal extension of the inner sum to $0<p<\infty$ can be made since the $a(\mu, m \cdot \mid p, \nu, n)$ will vanish for all the added terms. We can also extend the sum on $m$ to $(-\infty, \infty)$ since $P_{n}^{m}$ vanishes for all the added terms. But now, if we interchange the $p$ - and $n$-summations, and substitute a new index $t=\mu+m$, we obtain from Eq. (A2-1)

$$
\begin{gathered}
z_{\nu}(k R) P_{\nu}^{\mu}(\cos \theta) \exp (i \mu \phi)=i^{-\nu} \sum_{p=0}^{\infty} \sum_{i=-\infty}^{\infty} \sum_{n=0}^{\infty}\left\{i^{n+p}(2 n+1)(-)^{t-\mu} a(\mu, t-\mu \mid p, n, \nu)\right. \\
\cdot z_{p}\left(k r_{>}\right) P_{p}^{t}\left(\cos \theta_{>}\right) \exp \left(i t \phi_{>}\right) \\
\left.\cdot j_{n}\left(k r_{<}\right) P_{n}^{\mu-t}\left(\cos \theta_{<}\right) \exp \left[i(\mu-t) \phi_{<}\right]\right\} .
\end{gathered}
$$

If we now interchange the letters $p$ and $n$; write an $m$ for $t$; and also note that $P_{\mathfrak{x}}^{t}=0$ whenever $|t|>p$, we can rewrite the above as

$$
\begin{gathered}
z_{\nu}(k R) P_{\nu}^{\mu}(\cos \theta) \exp (i \mu \phi)=i^{-\nu} \sum_{n=0}^{\infty} \sum_{m=-n}^{\infty} \sum_{p=0}^{\infty}\left\{i^{n+p}(2 p+1)(-)^{m-\mu} a(\mu, m-\mu \mid n, p, \nu)\right. \\
\cdot z_{n}\left(k r_{>}\right) P_{n}^{m}\left(\cos \theta_{>}\right) \exp \left(i m \phi_{>}\right) \\
\left.\cdot j_{p}\left(k r_{<}\right) P_{p}^{\mu-m}\left(\cos \theta_{<}\right) \exp \left[i(\mu-m) \phi_{<}\right]\right\} .
\end{gathered}
$$


It is readily confirmed that the coefficient in this last equation also vanishes unless, at least, $n+\nu \geq p \geq|n-\nu|$, so that it is even more in a form similar to Eq. (A2-1). By now comparing with Eq. (A2-3), we see that for $R^{\prime} \geq R_{0}$, the result corresponding to Eq. (A2-4) is

$\alpha(\mu, \nu \mid m, n)=i^{-\nu+n}(-)^{m-\mu} \sum_{p} i^{p}(2 p+1) a(\mu, m-\mu \mid n, \nu, p) u_{p}^{\mu-m}\left(R_{0}, \theta_{0}, \phi_{0}\right)$,

where now $u_{p}^{\mu-m}$ contains explicitly the factor $j_{p}\left(k R_{0}\right)$ while the more general dependence on position has a $z_{n}\left(k R^{\prime}\right)$ of exactly the same cylinder function type as $z_{\nu}(k R)$.

Although this result appears to be different from the form in Eq. (A2-4), one expects on the basis of continuity of the two expansions across the surface $R^{\prime}=R_{0}$ that they should be equivalent. That this is indeed so, and hence that Eq. (A2-4) truly represents the required coefficient for all $R^{\prime}$, is most easily shown by referring to the more general representations of $a(\mu, m \mid p, \nu, n)$ coefficients as "vector-coupling coefficients" or "Wigner $3-j$ symbols". The equivalence can then be shown to follow from symmetry properties of the Wigner $3-j$ symbols [20].

Again special recurrence relations are available [21], while others can be derived from Eq. (A2-2) by using well-known Legendre function recurrence formulas.

\section{REFERENCES}

1. W. S. Ament, Wave propagation in suspensions, NRL Rept. 5307, April 9, 1959

2. J. Stratton, Electromagnetic theory, McGraw-Hill, N. Y., 1941, p. $414 \mathrm{ff}$.

3. Ibid., p. 404-406

4. Ibid., p. 401

5. R. Courant and D. Hilbert, Methods of mathematical physics, vol. 1, Interscience, N. Y., 1953, Appendix to Chap. 7, by W. Magnus

6. A. Erdelyi, et al., Higher transcendental functions, vol. 2, McGraw-Hill, N. Y., 1953 Chap. 11, esp. p. 257

7. Y. Sato, Transformation of wave-functions related to transformations of coordinate systems, Bull. Earthquake Research Inst. Tokyo 28, 1-22 and 175-217 (1950)

8. H. Hönl, Über ein Additionstheorem der Kugelfunktionen und seine Anwendung auf die Richtungsquantisierung der Atome, Z. Physik 89, 244-253 (1934)

9. A. Schmidt, Formeln zur Transformation der Kugelfunktionen bei linearer Änderung des Koordinatensystems, Z. Math. Phys. 44, 327-338 (1899)

10. H. McIntosh, A. Kleppner, and D. F. Minner, Tables of the Herglotz polynomials of orders $3 / 2,8 / 2$, transformation coefficients for spherical harmonics, BRL Memo., Rept. No. 1097, July 1957, Ballistic Research Laboratories, Aberdeen Proving Ground, Md.

11. A. R. Edmonds, Angular momentum in quantum mechanics, Princeton Univ. Press, Princeton, N. J., 1957, Chap. 4

12. Ibid., p. 24

13. Ibid., p. 6-8

14. Ibid., p. 61, Eq. 4.3.4, with $j_{1}=1$

15. J. S. Lamont, Applications of finite groups, Academic Press, N. Y., 1959, p. 150-151

16. Reference 11, p. 61, Eq. 4.3.2. with $j_{1}=1$

17. Reference 10, p. 21, in which the formulas are correct for the $U_{k l}^{n}$, rather than the $H_{k l}^{m}$ as written; also p. 19

18. B. Friedman and J. Russek, Addition theorems for spherical waves, Quart. Appl. Math. 12, 13-23 (1954)

19. E.g., A. Sommerfeld, Partial differential equations, Academic Press, N. Y., 1949, p. 128-129

20. Reference 11, p. 45 ff., also Eqs. 4.6.5, 3.7.5, 3.7.3, 3.6.10, 3.6.11, 3.1.5

21. Reference 11, p. $48-50$, and p. 95 